CRYSTALLOGRAPHIC COMMUNICATIONS

ISSN 2056-9890

Received 30 August 2021

Accepted 10 September 2021

Edited by O. Blacque, University of Zürich, Switzerland

Keywords: crystal structure; fluorene derivative; hydrogen bonding; Hirshfeld surface analysis; two-dimensional fingerprint plots.

CCDC reference: 2109160

Supporting information: this article has supporting information at journals.iucr.org/e

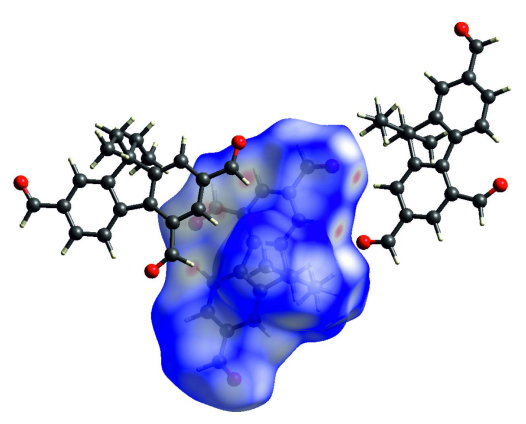

OPEN $\odot$ ACCESS

\section{Crystal structure of 9,9-diethyl-9H-fluorene-2,4,7- tricarbaldehyde}

\author{
Pierre Seidel, Anke Schwarzer and Monika Mazik*
}

Institut für Organische Chemie, Technische Universität Bergakademie Freiberg, Leipziger Str. 29, 09599 Freiberg, Germany. ${ }^{*}$ Correspondence e-mail: monika.mazik@chemie.tu-freiberg.de

The title compound, $\mathrm{C}_{20} \mathrm{H}_{18} \mathrm{O}_{3}$, crystallizes in the space group $P 2_{1} / c$ with one molecule in the asymmetric unit of the cell. The fluorene skeleton is nearly planar and the crystal structure is composed of molecular layers extending parallel to the (302) plane. Within a layer, one formyl oxygen atom participates in the formation of $\mathrm{C}_{\text {arene }}-\mathrm{H} \cdots \mathrm{O}$ bond, which is responsible for the formation of an inversion symmetric supramolecular motif of graph set $R_{2}^{2}(10)$. A second oxygen atom is involved in an intramolecular $\mathrm{C}_{\text {arene }}-\mathrm{H} \cdots \mathrm{O}$ hydrogen bond and is further connected with a formyl hydrogen atom of an adjacent molecule. A Hirshfeld surface analysis indicated that the most important contributions to the overall surface are from $\mathrm{H} \cdots \mathrm{H}(46.9 \%), \mathrm{O} \cdots \mathrm{H}(27.9 \%)$ and $\mathrm{C} \cdots \mathrm{H}(17.8 \%)$ interactions.

\section{Chemical context}

Compounds featuring a fluorene moiety have been recognized as useful for a broad spectrum of applications, which range from agents for cell imaging, solar cells, organic light-emitting diodes to lasers. Furthermore, fluorene derivatives have the potential to act as artificial receptors for different ionic and neutral substrates in analogy to the known receptors possessing a benzene or biphenyl core, which, for example, are able to complex ammonium ions (Koch et al., 2015; Schulze et al., 2018; Chin et al., 2002; Arunachalam et al., 2010), ion pairs (Stapf et al., 2015) or carbohydrates (Stapf et al., 2020; Köhler et al., 2020, 2021; Kaiser et al., 2019; Lippe \& Mazik, 2013, 2015; Amrhein et al., 2016; Amrhein \& Mazik, 2021). As a result of the manifold application possibilities of fluorenes, the syntheses of new representatives of this class of compounds are the subject of intensive research (Seidel et al., 2019, 2021; Seidel \& Mazik, 2020; Sicard et al., 2018). Fluorene derivatives bearing halogen, formyl or amino groups are valuable starting materials for a wide range of fluorene-based acyclic and macrocyclic compounds as well as polymers. Recently we have described the efficient one-step synthesis of 9,9-diethyl-9Hfluorene-2,4,7-tricarbaldehyde on the basis of 2,4,7-tris(bromomethyl)-9,9-diethyl-9H-fluorene (Seidel et al., 2019), which provided a threefold higher yield of the product than the previously known three-step reaction sequence (Yao \& Belfield, 2005). In this work we describe the crystal structure of this fluorene derivative bearing three formyl groups.

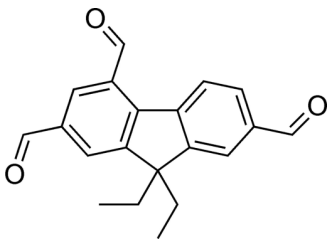


Table 1

Hydrogen-bond geometry $\left(\AA,^{\circ}\right)$.

\begin{tabular}{lllll}
\hline$D-\mathrm{H} \cdots A$ & $D-\mathrm{H}$ & $\mathrm{H} \cdots A$ & $D \cdots A$ & $D-\mathrm{H} \cdots A$ \\
\hline $\mathrm{C} 1-\mathrm{H} 1 \cdots \mathrm{O} 1^{\mathrm{i}}$ & 0.95 & 2.59 & $3.512(4)$ & 165 \\
$\mathrm{C} 5-\mathrm{H} 5 \cdots \mathrm{O} 2$ & 0.95 & 2.18 & $2.961(4)$ & 138 \\
$\mathrm{C} 5-\mathrm{H} 5 \cdots 3^{\mathrm{ii}}$ & 0.95 & 2.67 & $3.350(4)$ & 129 \\
$\mathrm{C} 16-\mathrm{H} 16 \cdots \mathrm{O} 2^{\mathrm{iii}}$ & 0.95 & 2.53 & $3.321(4)$ & 141 \\
$\mathrm{C} 17-\mathrm{H} 17 A \cdots \mathrm{O} 1^{\mathrm{i}}$ & 0.99 & 2.68 & $3.611(4)$ & 157 \\
\hline
\end{tabular}

Symmetry codes: (i) $\quad-x+1,-y+1,-z+1$; $\quad$ (ii) $\quad-x, y-\frac{1}{2},-z-\frac{1}{2}$; $\quad$ (iii) $-x, y+\frac{1}{2},-z-\frac{1}{2}$.

\section{Structural commentary}

The title compound (1) (Fig. 1) crystallizes in the space group $P 2_{1} / c$ with one molecule in the asymmetric unit. The 2,4,7substituted fluorene scaffold adopts a nearly planar geometry with the formyl groups inclined at angles of 4.2 (2), 3.5 (2) and $3.3(2)^{\circ}$ with respect to the fluorene moiety. These values correlate with torsion angles of $-175.8(3),-175.4$ (3) and $-176.7(4)^{\circ}$, respectively, for the atomic sequences $\mathrm{C} 3-\mathrm{C} 2-$ $\mathrm{C} 14-\mathrm{O} 1, \mathrm{C} 3-\mathrm{C} 4-\mathrm{C} 15-\mathrm{O} 2$ and $\mathrm{C} 6-\mathrm{C} 7-\mathrm{C} 16-\mathrm{O} 3$. The plane passing through the two ethyl groups is oriented nearly orthogonal to the plane of the fluorene unit [dihedral angle = $\left.89.8(1)^{\circ}\right]$. The oxygen atom $\mathrm{O} 2$ is involved in an intramolecular $\mathrm{C}_{\text {arene }}-\mathrm{H} \cdots \mathrm{O}$ hydrogen bond $[d(\mathrm{H} \cdots \mathrm{O}) 2.18 \AA$, $\mathrm{C}-\mathrm{H} \cdots \mathrm{O} 138^{\circ}$; Table 1].

\section{Supramolecular features}

The crystal structure of the title compound is composed of molecular layers extending parallel to the (302) plane. An excerpt of the layer structure showing the mode of hydrogen bonding is depicted in Fig. 2. Within a given layer, the formyl oxygen atom $\mathrm{O} 1$ participates in the formation of a $\mathrm{C}_{\text {arene }}-$ $\mathrm{H} \cdots \mathrm{O}$ bond $[d(\mathrm{H} \cdots \mathrm{O}) 2.59 \AA$; Table 1$]$, thus creating an inversion-symmetric supramolecular motif of graph-set $R_{2}^{2}(10)$ (Etter et al., 1990; Bernstein et al., 1995; for examples of other crystal structures including such a ten-membered supra-

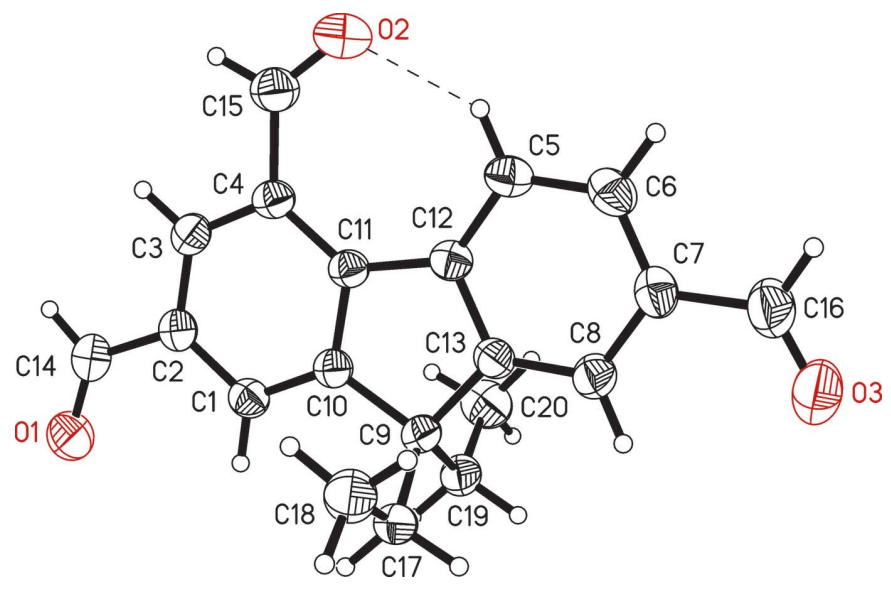

Figure 1

Perspective view of (1) including the labelling of non-hydrogen atoms. Displacement ellipsoids are drawn at the $50 \%$ probability level.

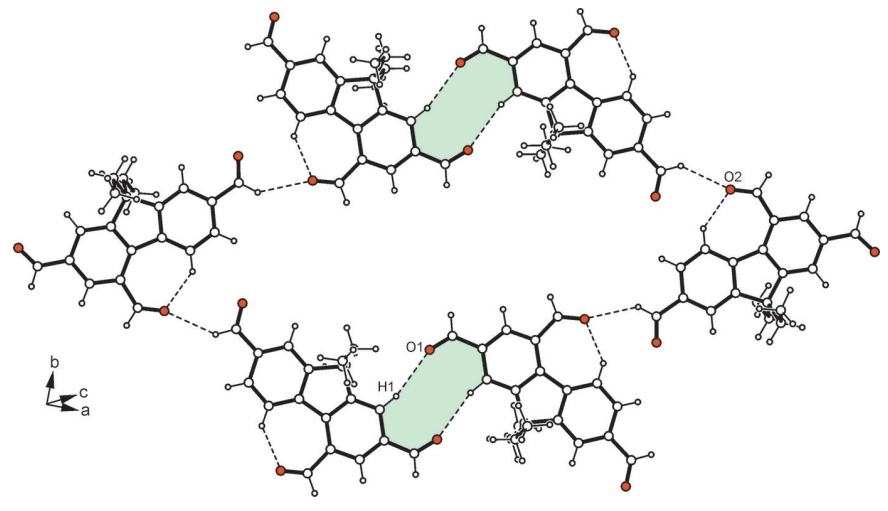

Figure 2

Packing excerpt of (1) showing selected $\mathrm{C}-\mathrm{H} \cdots \mathrm{O}$ interactions within one layer of molecules.

molecular motif, see Seidel et al., 2021; Stapf et al., 2021). The oxygen atom $\mathrm{O} 2$ is connected with the formyl hydrogen $\mathrm{H} 16$ of an adjacent molecule $[d(\mathrm{H} \cdots \mathrm{O}) 2.53 \AA]$. The steric requirements of the ethyl groups cause an offset of the molecules of consecutive layers, so that neither hydrogen bonds nor $\pi-\pi$ arene stacking interactions are observed in the direction of the layer normal. Consequently, the crystal appears to be stabilized by van der Waals forces in the direction of the stacking axis of the molecular layers (Fig. 3).

\section{Database Survey}

A search in the Cambridge Structural Database (Version 5.41, November 2019; Groom et al., 2016) for $9 H$-fluorene derivatives bearing a formyl group resulted in three hits, including $9 H$-fluorene carbaldehyde (SAZQIT; Dobson \& Gerkin, 1998) and two ferrocene-fluorene complexes including a 2-formyl-9-fluorenyl (HAPROF) and a 2,7-diformyl-9-fluorenyl moiety (HAPRUL; Wright \& Cochran, 1993). As in the case of the title compound, the $9 H$-fluorene carbaldehyde crystallized in the space group $P 2_{1} / c$ with one molecule in the asymmetric unit. The molecular core is nearly planar and the crystal structure is characterized by the presence of $\mathrm{C}-\mathrm{H} \cdots \mathrm{O}$ hydrogen bonds, which are responsible for the formation of a supramolecular motif of graph set $R_{2}^{2}(14)$.

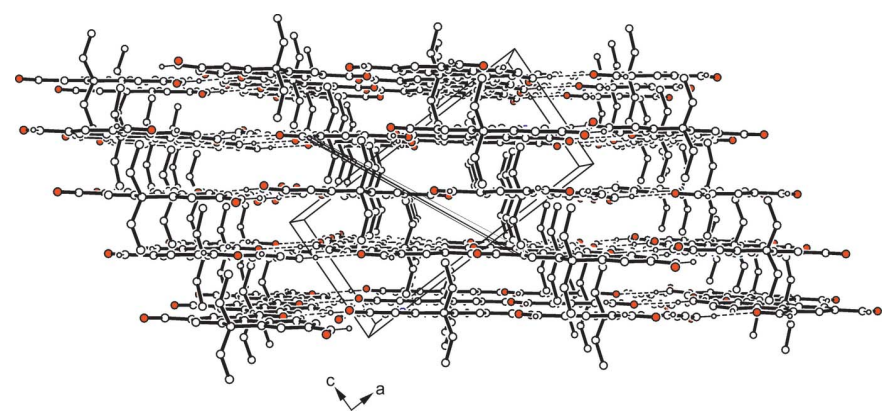

Figure 3

Packing excerpt of (1) showing adjacent layers of molecules and selected $\mathrm{C}-\mathrm{H} \cdots \mathrm{O}$ interactions within the layers. Hydrogen atoms of subunits not involved in intermolecular hydrogen bonding are omitted for clarity. 

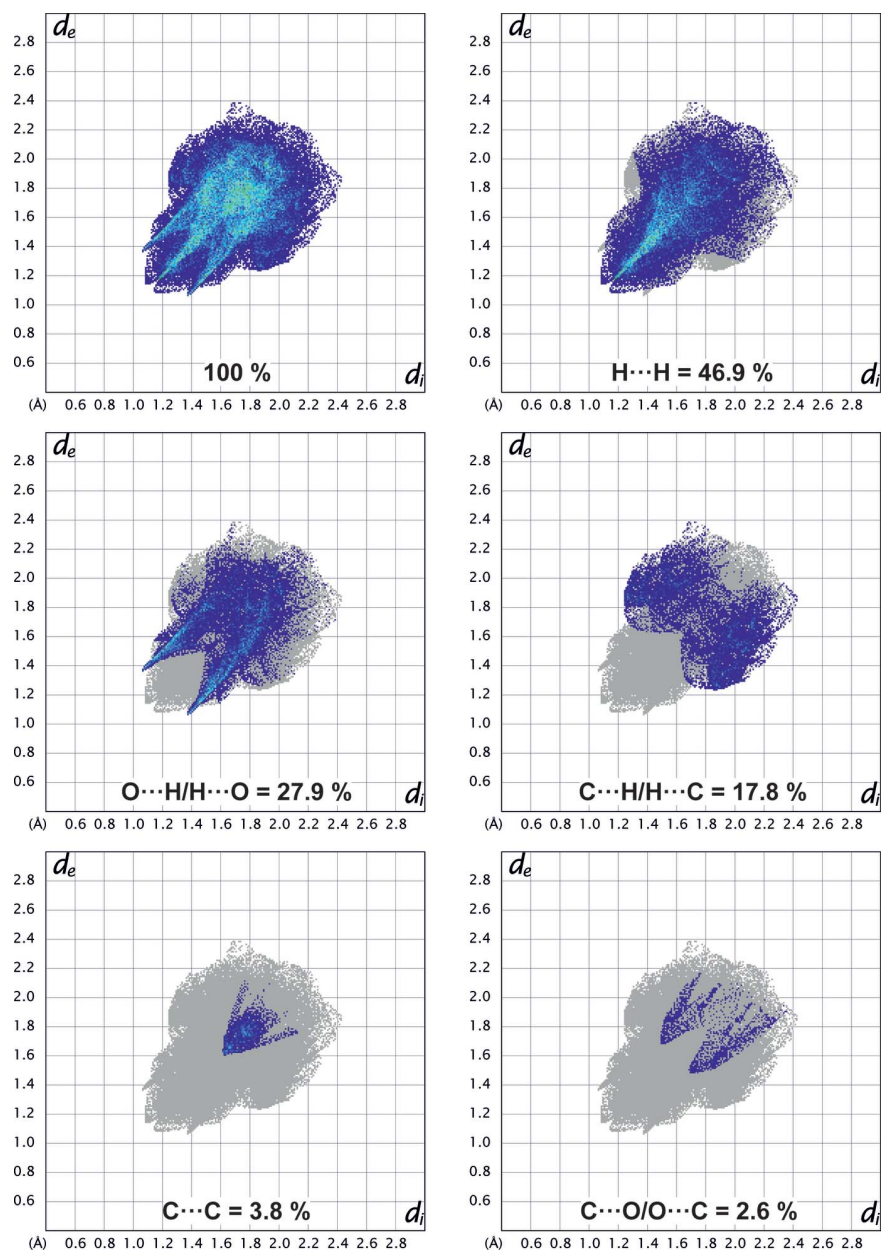

Figure 4

Fingerprint plot of (1) including the contribution of the atom $\cdots$ atom pairs to the overall surface.

\section{Hirshfeld surface analysis}

Hirshfeld surfaces (Spackman \& Jayatilaka, 2009) were calculated and the associated 2D fingerprint plots generated using Crystal Explorer 17.5 (Turner et al., 2017). The 2D fingerprint plots (McKinnon et al., 2007) are displayed within the expanded $0.4-3.0 \AA$ range including reciprocal contacts (Fig. 4); 3D $d_{\text {norm }}$ surfaces are mapped over a fixed colour scale of -0.3 a.u. (red)-1.0 a.u. (blue) (Figs. 5 and 6). The 2D fingerprint plots (see Fig. 4) indicate that the most important contributions to the overall surface are from $\mathrm{H} \cdots \mathrm{H}(46.9 \%)$,
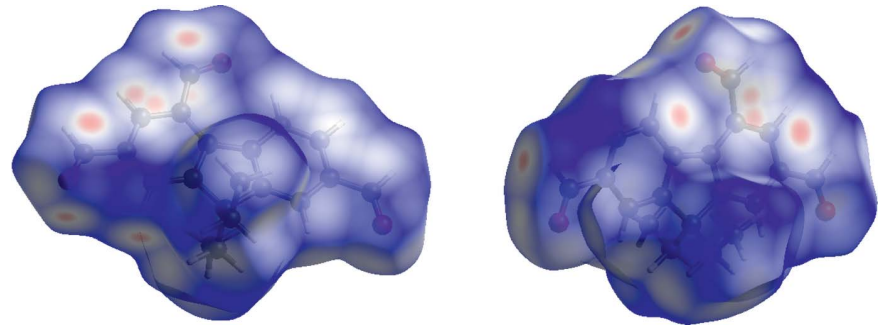

Figure 5

Hirshfeld surface for (1) mapped with $d_{\text {norm }}$ (front and back views).

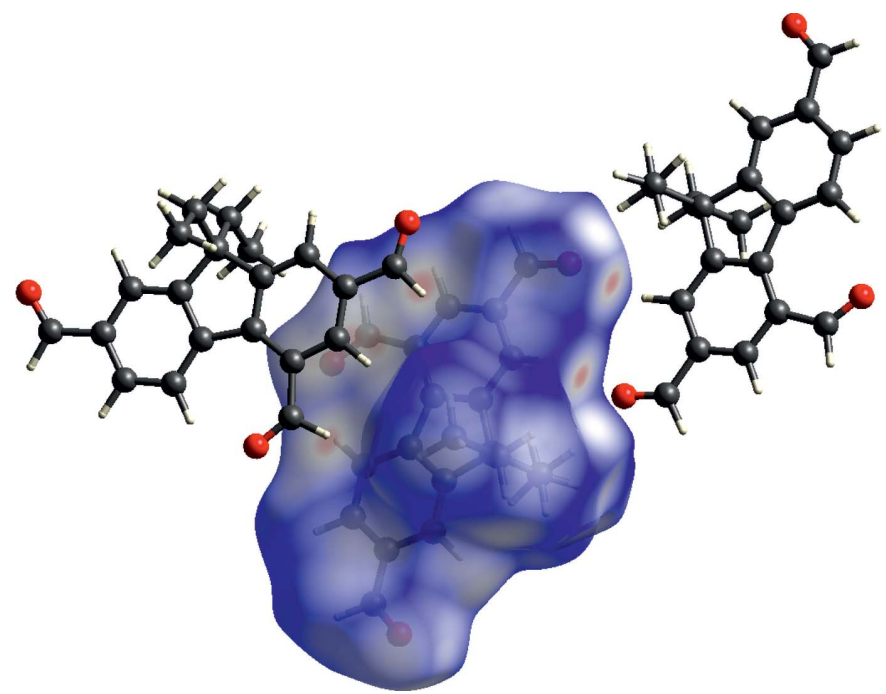

Figure 6

Hirshfeld surface for (1) mapped with $d_{\text {norm }}$ and shape-index function showing neigbouring molecules and the corresponding contacts.

$\mathrm{O} \cdots \mathrm{H}(27.9 \%)$ and $\mathrm{C} \cdot \mathrm{H}(17.8 \%)$ interactions, whereas only $3.8 \%$ and $2.6 \%$ are from the $\mathrm{C} \cdots \mathrm{C}$ and $\mathrm{C} \cdot \mathrm{O}$. contacts, respectively. In addition to the fingerprint plots, the Hirshfeld plots mapped with $d_{\text {norm }}$ give a hint about the significance of the close contacts. For example, the $\mathrm{O} \cdots \mathrm{H}$ hydrogen bonds are responsible for the intense red spots on the surface, as shown in Figs. 5 and 6.

\section{Synthesis and crystallization}

The title compound was prepared by an efficient one-step synthesis involving the treatment of 2,4,7-tris(bromomethyl)9,9-diethyl-9H-fluorene with $\mathrm{N}$-methylmorpholine $\mathrm{N}$-oxide (Seidel et al., 2019). Single crystals of (1) were achieved via crystallization from a mixture of dichloromethane and $n$ hexane $(1: 1 v / v)$.

\section{Refinement}

Crystal data, data collection and structure refinement details are summarized in Table 2. The non-hydrogen atoms were refined anisotropically. All hydrogen atoms were positioned geometrically and allowed to ride on their parent atoms: $\mathrm{C}-\mathrm{H}$ $=0.95 \AA$ for aryl-H atoms, $\mathrm{C}-\mathrm{H}=0.99 \AA$ for methylene groups and $\mathrm{C}-\mathrm{H}=0.98 \AA$ for methyl groups with $U_{\text {iso }}(\mathrm{H})=$ $1.5 U_{\text {eq }}(\mathrm{C})$ for methyl groups and $U_{\text {iso }}(\mathrm{H})=1.2 U_{\text {eq }}(\mathrm{C})$ for other hydrogen atoms. The crystal structure of (1) was refined as a two-component twin with an approximate occupancy ratio of 63:37.

\section{Acknowledgements}

Open Access Funding by the Publication Fund of the Technische Universität Bergakademie Freiberg is gratefully acknowledged. 
Table 2

Experimental details.

\begin{tabular}{|c|c|}
\hline \multicolumn{2}{|l|}{ Crystal data } \\
\hline Chemical formula & $\mathrm{C}_{20} \mathrm{H}_{18} \mathrm{O}_{3}$ \\
\hline$M_{\mathrm{r}}$ & 306.34 \\
\hline Crystal system, space group & Monoclinic, $P 2_{1} / c$ \\
\hline Temperature $(\mathrm{K})$ & 150 \\
\hline$a, b, c(\AA)$ & $\begin{array}{l}15.6595(9), 13.1466(14), \\
\quad 7.6834(15)\end{array}$ \\
\hline$\beta\left(^{\circ}\right)$ & $93.146(9)$ \\
\hline$V\left(\AA^{3}\right)$ & $1579.4(4)$ \\
\hline$Z$ & 4 \\
\hline Radiation type & Мо $K \alpha$ \\
\hline$\mu\left(\mathrm{mm}^{-1}\right)$ & 0.09 \\
\hline Crystal size $(\mathrm{mm})$ & $0.38 \times 0.30 \times 0.15$ \\
\hline \multicolumn{2}{|l|}{ Data collection } \\
\hline Diffractometer & Stoe IPDS $2 \mathrm{~T}$ \\
\hline Absorption correction & - \\
\hline $\begin{array}{l}\text { No. of measured, independent and } \\
\text { observed }[I>2 \sigma(I)] \text { reflections }\end{array}$ & $13951,13951,8830$ \\
\hline$R_{\text {int }}$ & $?$ \\
\hline$(\sin \theta / \lambda)_{\max }\left(\AA^{-1}\right)$ & 0.594 \\
\hline \multicolumn{2}{|l|}{ Refinement } \\
\hline$R\left[F^{2}>2 \sigma\left(F^{2}\right)\right], w R\left(F^{2}\right), S$ & $0.047,0.127,0.93$ \\
\hline No. of reflections & 13951 \\
\hline No. of parameters & 211 \\
\hline $\mathrm{H}$-atom treatment & $\mathrm{H}$-atom parameters constrained \\
\hline$\Delta \rho_{\max }, \Delta \rho_{\min }\left(\mathrm{e} \AA^{-3}\right)$ & $0.26,-0.24$ \\
\hline
\end{tabular}

Computer programs: $X$-AREA and X-RED (Stoe, 2009), SHELXT2018/2 (Sheldrick, 2015a), SHELXL2018/3 (Sheldrick, 2015b), XP (Sheldrick, 2008), WinGX (Farrugia, 2012), publCIF (Westrip, 2010) and shelXle (Hübschle et al., 2011).

\section{References}

Amrhein, F., Lippe, J. \& Mazik, M. (2016). Org. Biomol. Chem. 14, 10648-10659.

Amrhein, F. \& Mazik, M. (2021). Eur. J. Org. Chem. https://doi.org/ 10.1002/ejoc.202100758

Arunachalam, M., Ahamed, B. N. \& Ghosh, P. (2010). Org. Lett. 12, 2742-2745.

Bernstein, J., Davis, R. E., Shimoni, L. \& Chang, N.-L. (1995). Angew. Chem. Int. Ed. Engl. 34, 1555-1573.

Chin, J., Oh, J., Jon, S. Y., Park, S. H., Walsdorff, C., Stranix, B., Ghoussoub, A., Lee, S. J., Chung, H. J., Park, S.-M. \& Kim, K. (2002). J. Am. Chem. Soc. 124, 5374-5379.

Dobson, A. J. \& Gerkin, R. E. (1998). Acta Cryst. C54, 1890-1892.
Etter, M. C., MacDonald, J. C. \& Bernstein, J. (1990). Acta Cryst. B46, 256-262.

Farrugia, L. J. (2012). J. Appl. Cryst. 45, 849-854.

Groom, C. R., Bruno, I. J., Lightfoot, M. P. \& Ward, S. C. (2016). Acta Cryst. B72, 171-179.

Hübschle, C. B., Sheldrick, G. M. \& Dittrich, B. (2011). J. Appl. Cryst. 44, 1281-1284.

Kaiser, S., Geffert, C. \& Mazik, M. (2019). Eur. J. Org. Chem. pp. 7555-7562.

Koch, N., Seichter, W. \& Mazik, M. (2015). Tetrahedron, 71, 89658974.

Köhler, L., Hübler, C., Seichter, W. \& Mazik, M. (2021). RSC Adv. 11, 22221-22229.

Köhler, L., Seichter, W. \& Mazik, M. (2020). Eur. J. Org. Chem. pp. 7023-7034.

Lippe, J. \& Mazik, M. (2013). J. Org. Chem. 78, 9013-9020.

Lippe, J. \& Mazik, M. (2015). J. Org. Chem. 80, 1427-1439.

McKinnon, J. J., Jayatilaka, D. \& Spackman, M. A. (2007). Chem. Commun. pp. 3814-3816.

Schulze, M., Koch, N., Seichter, W. \& Mazik, M. (2018). Eur. J. Org. Chem. pp. 4317-4330.

Seidel, P. \& Mazik, M. (2020). ChemistryOpen, 9, 1202-1213.

Seidel, P., Schwarzer, A. \& Mazik, M. (2019). Eur. J. Org. Chem. pp. 1493-1502.

Seidel, P., Seichter, W., Schwarzer, A. \& Mazik, M. (2021). Eur. J. Org. Chem. pp. 2901-2914.

Sheldrick, G. M. (2008). Acta Cryst. A64, 112-122.

Sheldrick, G. M. (2015a). Acta Cryst. A71, 3-8.

Sheldrick, G. M. (2015b). Acta Cryst. C71, 3-8.

Sicard, L., Jeannin, O., Rault-Berthelot, J., Quinton, C. \& Poriel, C. (2018). ChemPlusChem, 83, 874-880.

Spackman, M. A. \& Jayatilaka, D. (2009). CrystEngComm, 11, 19-32.

Stapf, M., Leibiger, B., Schwarzer, A. \& Mazik, M. (2021). Acta Cryst. E77, 919-923.

Stapf, M., Seichter, W. \& Mazik, M. (2015). Chem. Eur. J. 21, 63506354.

Stapf, M., Seichter, W. \& Mazik, M. (2020). Eur. J. Org. Chem. 2020, 4900-4915.

Stoe (2009). $X$-RED and $X$-AREA. Stoe \& Cie, Darmstadt, Germany.

Turner, M. J., McKinnon, J. J., Wolff, S. K., Grimwood, D. J., Spackman, P. R., Jayatilaka, D. \& Spackman, M. A. (2017). CrystalExplorer17. The University of Western Australia.

Westrip, S. P. (2010). J. Appl. Cryst. 43, 920-925.

Wright, M. E. \& Cochran, B. B. (1993). Organometallics, 12, $3873-$ 3878.

Yao, S. \& Belfield, K. D. (2005). J. Org. Chem. 70, 5126-5132. 


\section{supporting information}

Acta Cryst. (2021). E77, 1029-1032［https://doi.org/10.1107/S2056989021009464]

\section{Crystal structure of 9,9-diethyl-9H-fluorene-2,4,7-tricarbaldehyde}

\section{Pierre Seidel, Anke Schwarzer and Monika Mazik}

\section{Computing details}

Data collection: $X$-AREA (Stoe, 2009); cell refinement: $X$-AREA (Stoe, 2009); data reduction: $X$-RED (Stoe, 2009); program(s) used to solve structure: SHELXT2018/2 (Sheldrick, 2015a); program(s) used to refine structure:

SHELXL2018/3 (Sheldrick, 2015b); molecular graphics: XP (Sheldrick, 2008); software used to prepare material for publication: WinGX (Farrugia, 2012), publCIF (Westrip, 2010), and shelXle (Hübschle et al., 2011).

9,9-Diethylfluorene-2,4,7-tricarbaldehyde

\section{Crystal data}

$\mathrm{C}_{20} \mathrm{H}_{18} \mathrm{O}_{3}$

$M_{r}=306.34$

Monoclinic, $P 2{ }_{1} / c$

$a=15.6595(9) \AA$

$b=13.1466(14) \AA$

$c=7.6834(15) \AA$

$\beta=93.146(9)^{\circ}$

$V=1579.4(4) \AA^{3}$

$Z=4$

Data collection

Stoe IPDS 2T diffractometer

Radiation source: sealed X-ray tube, 12 x 0.4 $\mathrm{mm}$ long-fine focus

Plane graphite monochromator

Detector resolution: 6.67 pixels $\mathrm{mm}^{-1}$

rotation method scans

\section{Refinement}

Refinement on $F^{2}$

Least-squares matrix: full

$R\left[F^{2}>2 \sigma\left(F^{2}\right)\right]=0.047$

$w R\left(F^{2}\right)=0.127$

$S=0.93$

13951 reflections

211 parameters

0 restraints
$F(000)=648$

$D_{\mathrm{x}}=1.288 \mathrm{Mg} \mathrm{m}^{-3}$

Mo $K \alpha$ radiation, $\lambda=0.71073 \AA$

Cell parameters from 6293 reflections

$\theta=2.9-28.3^{\circ}$

$\mu=0.09 \mathrm{~mm}^{-1}$

$T=150 \mathrm{~K}$

Piece, colorless

$0.38 \times 0.30 \times 0.15 \mathrm{~mm}$

13951 measured reflections 13951 independent reflections 8830 reflections with $I>2 \sigma(I)$

$\theta_{\max }=25.0^{\circ}, \theta_{\min }=3.0^{\circ}$

$h=-17 \rightarrow 18$

$k=-15 \rightarrow 15$

$l=-9 \rightarrow 9$

Hydrogen site location: inferred from neighbouring sites

$\mathrm{H}$-atom parameters constrained

$w=1 /\left[\sigma^{2}\left(F_{\mathrm{o}}{ }^{2}\right)+(0.0713 P)^{2}\right]$

where $P=\left(F_{\mathrm{o}}^{2}+2 F_{\mathrm{c}}^{2}\right) / 3$

$(\Delta / \sigma)_{\max }<0.001$

$\Delta \rho_{\max }=0.26 \mathrm{e}^{-3}$

$\Delta \rho_{\min }=-0.24$ e $\AA^{-3}$ 


\section{Special details}

Geometry. All esds (except the esd in the dihedral angle between two 1.s. planes) are estimated using the full covariance matrix. The cell esds are taken into account individually in the estimation of esds in distances, angles and torsion angles; correlations between esds in cell parameters are only used when they are defined by crystal symmetry. An approximate (isotropic) treatment of cell esds is used for estimating esds involving l.s. planes.

Refinement. Refined as a two-component twin.

Fractional atomic coordinates and isotropic or equivalent isotropic displacement parameters $\left(\AA^{2}\right)$

\begin{tabular}{|c|c|c|c|c|}
\hline & $x$ & $y$ & $z$ & $U_{\text {iso }} * / U_{\text {eq }}$ \\
\hline $\mathrm{O} 1$ & 0.51535 (14) & $0.34892(18)$ & 0.5435 (3) & $0.0532(7)$ \\
\hline $\mathrm{O} 2$ & $0.15641(16)$ & 0.18707 (17) & $-0.0059(3)$ & $0.0604(7)$ \\
\hline $\mathrm{O} 3$ & $0.03712(16)$ & $0.7414(2)$ & $-0.2592(4)$ & $0.0713(8)$ \\
\hline $\mathrm{C} 1$ & $0.37790(18)$ & $0.4293(2)$ & $0.3116(3)$ & $0.0322(7)$ \\
\hline H1 & 0.414572 & 0.480263 & 0.361567 & $0.039 *$ \\
\hline $\mathrm{C} 2$ & $0.39372(18)$ & $0.3258(2)$ & $0.3466(3)$ & $0.0316(7)$ \\
\hline $\mathrm{C} 3$ & 0.33905 (17) & $0.2531(2)$ & $0.2722(4)$ & $0.0330(6)$ \\
\hline $\mathrm{H} 3$ & 0.350875 & 0.183387 & 0.295850 & $0.040^{*}$ \\
\hline $\mathrm{C} 4$ & $0.26770(17)$ & $0.2774(2)$ & $0.1647(3)$ & $0.0313(7)$ \\
\hline $\mathrm{C} 5$ & $0.11308(18)$ & $0.4025(2)$ & $-0.0802(4)$ & $0.0372(7)$ \\
\hline H5 & 0.099564 & 0.332138 & -0.087467 & $0.045^{*}$ \\
\hline C6 & $0.06274(19)$ & $0.4738(2)$ & $-0.1701(4)$ & $0.0412(8)$ \\
\hline H6 & 0.014947 & 0.451369 & -0.241394 & $0.049 *$ \\
\hline $\mathrm{C} 7$ & $0.08030(19)$ & $0.5775(2)$ & $-0.1586(4)$ & $0.0385(7)$ \\
\hline C8 & $0.15063(18)$ & $0.6120(2)$ & $-0.0543(4)$ & $0.0364(7)$ \\
\hline H8 & 0.162721 & 0.682613 & -0.044365 & $0.044^{*}$ \\
\hline $\mathrm{C} 9$ & $0.28194(18)$ & $0.5627(2)$ & $0.1500(4)$ & $0.0313(7)$ \\
\hline $\mathrm{C} 10$ & $0.30798(17)$ & $0.4554(2)$ & $0.2032(3)$ & $0.0294(6)$ \\
\hline C11 & $0.25144(17)$ & $0.3818(2)$ & $0.1286(3)$ & $0.0291(6)$ \\
\hline $\mathrm{C} 12$ & $0.18436(17)$ & $0.4364(2)$ & 0.0215 (3) & $0.0305(6)$ \\
\hline $\mathrm{C} 13$ & $0.20206(18)$ & $0.5414(2)$ & 0.0338 (3) & $0.0311(7)$ \\
\hline $\mathrm{C} 14$ & $0.46610(18)$ & $0.2932(2)$ & 0.4635 (4) & $0.0379(7)$ \\
\hline H14 & 0.474848 & 0.222039 & 0.477297 & $0.045^{*}$ \\
\hline $\mathrm{C} 15$ & $0.2180(2)$ & $0.1882(2)$ & 0.0975 (4) & $0.0436(8)$ \\
\hline H15 & 0.236754 & 0.123771 & 0.140831 & $0.052 *$ \\
\hline $\mathrm{C} 16$ & $0.0245(2)$ & $0.6512(3)$ & $-0.2560(5)$ & $0.0535(9)$ \\
\hline H16 & -0.024467 & 0.625546 & -0.320103 & $0.064^{*}$ \\
\hline $\mathrm{C} 17$ & $0.26251(19)$ & $0.6290(2)$ & $0.3086(4)$ & $0.0366(7)$ \\
\hline H17A & 0.315855 & 0.636730 & 0.382697 & $0.044^{*}$ \\
\hline H17B & 0.245393 & 0.697499 & 0.266210 & $0.044^{*}$ \\
\hline C18 & 0.1931 (2) & $0.5885(2)$ & $0.4212(4)$ & $0.0466(8)$ \\
\hline H18A & 0.190690 & 0.630388 & 0.526375 & $0.070^{*}$ \\
\hline H18B & 0.206083 & 0.518019 & 0.454380 & $0.070^{*}$ \\
\hline $\mathrm{H} 18 \mathrm{C}$ & 0.137719 & 0.591187 & 0.355334 & $0.070^{*}$ \\
\hline C19 & $0.35203(19)$ & $0.6155(2)$ & 0.0494 (4) & $0.0388(7)$ \\
\hline H19A & 0.331460 & 0.684240 & 0.015221 & $0.047^{*}$ \\
\hline H19B & 0.403150 & 0.624283 & 0.129659 & $0.047 *$ \\
\hline $\mathrm{C} 20$ & $0.3791(2)$ & $0.5611(3)$ & $-0.1127(4)$ & $0.0516(9)$ \\
\hline
\end{tabular}


supporting information

\begin{tabular}{lllll}
$\mathrm{H} 20 \mathrm{~A}$ & 0.419668 & 0.603613 & -0.172536 & $0.077^{*}$ \\
$\mathrm{H} 20 \mathrm{~B}$ & 0.328650 & 0.548144 & -0.190883 & $0.077^{*}$ \\
$\mathrm{H} 20 \mathrm{C}$ & 0.406310 & 0.496296 & -0.079554 & $0.077^{*}$ \\
\hline
\end{tabular}

Atomic displacement parameters $\left(\AA^{2}\right)$

\begin{tabular}{lllllll}
\hline & $U^{11}$ & $U^{22}$ & $U^{33}$ & $U^{12}$ & $U^{13}$ & $U^{23}$ \\
\hline O1 & $0.0435(13)$ & $0.0551(15)$ & $0.0591(14)$ & $-0.0035(12)$ & $-0.0151(13)$ & $0.0044(13)$ \\
O2 & $0.0656(15)$ & $0.0508(15)$ & $0.0633(15)$ & $-0.0157(12)$ & $-0.0094(14)$ & $-0.0051(12)$ \\
O3 & $0.0680(16)$ & $0.0551(16)$ & $0.0880(18)$ & $0.0021(15)$ & $-0.0219(16)$ & $0.0182(15)$ \\
C1 & $0.0309(15)$ & $0.0348(16)$ & $0.0311(13)$ & $-0.0035(13)$ & $0.0026(13)$ & $-0.0008(12)$ \\
C2 & $0.0302(15)$ & $0.0353(16)$ & $0.0296(15)$ & $0.0015(13)$ & $0.0045(13)$ & $0.0014(12)$ \\
C3 & $0.0389(15)$ & $0.0300(15)$ & $0.0308(14)$ & $0.0026(14)$ & $0.0072(14)$ & $0.0007(12)$ \\
C4 & $0.0349(15)$ & $0.0303(16)$ & $0.0292(14)$ & $-0.0029(13)$ & $0.0071(14)$ & $-0.0032(12)$ \\
C5 & $0.0341(16)$ & $0.0363(16)$ & $0.0409(16)$ & $-0.0056(14)$ & $0.0000(14)$ & $-0.0041(14)$ \\
C6 & $0.0320(17)$ & $0.049(2)$ & $0.0421(17)$ & $-0.0048(15)$ & $-0.0037(15)$ & $-0.0012(14)$ \\
C7 & $0.0332(16)$ & $0.0450(19)$ & $0.0371(15)$ & $0.0006(14)$ & $-0.0003(14)$ & $0.0057(14)$ \\
C8 & $0.0360(16)$ & $0.0350(16)$ & $0.0381(16)$ & $-0.0038(14)$ & $0.0003(14)$ & $0.0044(13)$ \\
C9 & $0.0330(15)$ & $0.0263(14)$ & $0.0344(14)$ & $-0.0022(12)$ & $-0.0008(13)$ & $0.0009(12)$ \\
C10 & $0.0303(15)$ & $0.0301(15)$ & $0.0280(13)$ & $-0.0010(13)$ & $0.0034(13)$ & $-0.0004(12)$ \\
C11 & $0.0314(15)$ & $0.0289(15)$ & $0.0274(13)$ & $-0.0020(13)$ & $0.0042(12)$ & $-0.0018(12)$ \\
C12 & $0.0287(15)$ & $0.0321(15)$ & $0.0309(14)$ & $-0.0024(12)$ & $0.0036(13)$ & $-0.0014(13)$ \\
C13 & $0.0316(15)$ & $0.0328(15)$ & $0.0288(13)$ & $-0.0030(13)$ & $0.0001(13)$ & $-0.0002(13)$ \\
C14 & $0.0355(16)$ & $0.0414(17)$ & $0.0371(17)$ & $0.0051(15)$ & $0.0056(15)$ & $0.0057(15)$ \\
C15 & $0.050(2)$ & $0.0422(19)$ & $0.0389(17)$ & $-0.0085(16)$ & $0.0045(17)$ & $-0.0019(15)$ \\
C16 & $0.044(2)$ & $0.055(2)$ & $0.060(2)$ & $-0.0005(18)$ & $-0.0105(18)$ & $0.0115(19)$ \\
C17 & $0.0403(17)$ & $0.0308(15)$ & $0.0378(15)$ & $0.0010(14)$ & $-0.0054(14)$ & $-0.0035(13)$ \\
C18 & $0.0519(19)$ & $0.0454(19)$ & $0.0427(17)$ & $0.0051(16)$ & $0.0043(16)$ & $-0.0022(15)$ \\
C19 & $0.0378(16)$ & $0.0349(16)$ & $0.0430(16)$ & $-0.0097(13)$ & $-0.0046(15)$ & $0.0071(14)$ \\
C20 & $0.0473(19)$ & $0.060(2)$ & $0.0478(18)$ & $-0.0153(18)$ & $0.0095(16)$ & $0.0028(17)$ \\
& & & & & & \\
\hline
\end{tabular}

Geometric parameters $\left(\AA,{ }^{\circ}\right)$

\begin{tabular}{llll}
\hline $\mathrm{O} 1-\mathrm{C} 14$ & $1.207(3)$ & $\mathrm{C} 9-\mathrm{C} 13$ & $1.523(4)$ \\
$\mathrm{O} 2-\mathrm{C} 15$ & $1.216(3)$ & $\mathrm{C} 9-\mathrm{C} 19$ & $1.542(4)$ \\
$\mathrm{O} 3-\mathrm{C} 16$ & $1.203(4)$ & $\mathrm{C} 9-\mathrm{C} 17$ & $1.542(4)$ \\
$\mathrm{C} 1-\mathrm{C} 10$ & $1.382(4)$ & $\mathrm{C} 10-\mathrm{C} 11$ & $1.411(4)$ \\
$\mathrm{C} 1-\mathrm{C} 2$ & $1.406(4)$ & $\mathrm{C} 11-\mathrm{C} 12$ & $1.483(4)$ \\
$\mathrm{C} 1-\mathrm{H} 1$ & 0.9500 & $\mathrm{C} 12-\mathrm{C} 13$ & $1.410(4)$ \\
$\mathrm{C} 2-\mathrm{C} 3$ & $1.386(4)$ & $\mathrm{C} 14-\mathrm{H} 14$ & 0.9500 \\
$\mathrm{C} 2-\mathrm{C} 14$ & $1.471(4)$ & $\mathrm{C} 15-\mathrm{H} 15$ & 0.9500 \\
$\mathrm{C} 3-\mathrm{C} 4$ & $1.390(4)$ & $\mathrm{C} 16-\mathrm{H} 16$ & 0.9500 \\
$\mathrm{C} 3-\mathrm{H} 3$ & 0.9500 & $\mathrm{C} 17-\mathrm{C} 18$ & $1.522(5)$ \\
$\mathrm{C} 4-\mathrm{C} 11$ & $1.421(4)$ & $\mathrm{C} 17-\mathrm{H} 17 \mathrm{~A}$ & 0.9900 \\
$\mathrm{C} 4-\mathrm{C} 15$ & $1.484(4)$ & $\mathrm{C} 17-\mathrm{H} 17 \mathrm{~B}$ & 0.9900 \\
$\mathrm{C} 5-\mathrm{C} 6$ & $1.384(4)$ & $\mathrm{C} 18-\mathrm{H} 18 \mathrm{~A}$ & 0.9800 \\
$\mathrm{C} 5-\mathrm{C} 12$ & $1.400(4)$ & $\mathrm{C} 18-\mathrm{H} 18 \mathrm{~B}$ & 0.9800 \\
$\mathrm{C} 5-\mathrm{H} 5$ & 0.9500 & $\mathrm{C} 18-\mathrm{H} 18 \mathrm{C}$ & 0.9800
\end{tabular}




\begin{tabular}{|c|c|c|c|}
\hline $\mathrm{C} 6-\mathrm{C} 7$ & $1.393(4)$ & $\mathrm{C} 19-\mathrm{C} 20$ & $1.517(5)$ \\
\hline $\mathrm{C} 6-\mathrm{H} 6$ & 0.9500 & $\mathrm{C} 19-\mathrm{H} 19 \mathrm{~A}$ & 0.9900 \\
\hline $\mathrm{C} 7-\mathrm{C} 8$ & $1.401(4)$ & C19-H19B & 0.9900 \\
\hline $\mathrm{C} 7-\mathrm{C} 16$ & $1.479(4)$ & $\mathrm{C} 20-\mathrm{H} 20 \mathrm{~A}$ & 0.9800 \\
\hline $\mathrm{C} 8-\mathrm{C} 13$ & $1.381(4)$ & $\mathrm{C} 20-\mathrm{H} 20 \mathrm{~B}$ & 0.9800 \\
\hline $\mathrm{C} 8-\mathrm{H} 8$ & 0.9500 & $\mathrm{C} 20-\mathrm{H} 20 \mathrm{C}$ & 0.9800 \\
\hline $\mathrm{C} 9-\mathrm{C} 10$ & $1.518(4)$ & & \\
\hline $\mathrm{C} 10-\mathrm{C} 1-\mathrm{C} 2$ & $118.8(2)$ & $\mathrm{C} 13-\mathrm{C} 12-\mathrm{C} 11$ & $107.8(2)$ \\
\hline $\mathrm{C} 10-\mathrm{C} 1-\mathrm{H} 1$ & 120.6 & $\mathrm{C} 8-\mathrm{C} 13-\mathrm{C} 12$ & $121.2(2)$ \\
\hline $\mathrm{C} 2-\mathrm{C} 1-\mathrm{H} 1$ & 120.6 & $\mathrm{C} 8-\mathrm{C} 13-\mathrm{C} 9$ & $127.0(2)$ \\
\hline $\mathrm{C} 3-\mathrm{C} 2-\mathrm{C} 1$ & $119.4(2)$ & $\mathrm{C} 12-\mathrm{C} 13-\mathrm{C} 9$ & $111.8(2)$ \\
\hline $\mathrm{C} 3-\mathrm{C} 2-\mathrm{C} 14$ & $119.2(3)$ & $\mathrm{O} 1-\mathrm{C} 14-\mathrm{C} 2$ & $125.7(3)$ \\
\hline $\mathrm{C} 1-\mathrm{C} 2-\mathrm{C} 14$ & $121.4(2)$ & $\mathrm{O} 1-\mathrm{C} 14-\mathrm{H} 14$ & 117.2 \\
\hline $\mathrm{C} 2-\mathrm{C} 3-\mathrm{C} 4$ & $123.0(3)$ & $\mathrm{C} 2-\mathrm{C} 14-\mathrm{H} 14$ & 117.2 \\
\hline $\mathrm{C} 2-\mathrm{C} 3-\mathrm{H} 3$ & 118.5 & $\mathrm{O} 2-\mathrm{C} 15-\mathrm{C} 4$ & $128.2(3)$ \\
\hline $\mathrm{C} 4-\mathrm{C} 3-\mathrm{H} 3$ & 118.5 & $\mathrm{O} 2-\mathrm{C} 15-\mathrm{H} 15$ & 115.9 \\
\hline $\mathrm{C} 3-\mathrm{C} 4-\mathrm{C} 11$ & $117.9(2)$ & $\mathrm{C} 4-\mathrm{C} 15-\mathrm{H} 15$ & 115.9 \\
\hline $\mathrm{C} 3-\mathrm{C} 4-\mathrm{C} 15$ & $114.4(3)$ & $\mathrm{O} 3-\mathrm{C} 16-\mathrm{C} 7$ & $124.2(3)$ \\
\hline $\mathrm{C} 11-\mathrm{C} 4-\mathrm{C} 15$ & $127.7(2)$ & $\mathrm{O} 3-\mathrm{C} 16-\mathrm{H} 16$ & 117.9 \\
\hline $\mathrm{C} 6-\mathrm{C} 5-\mathrm{C} 12$ & $118.5(3)$ & $\mathrm{C} 7-\mathrm{C} 16-\mathrm{H} 16$ & 117.9 \\
\hline $\mathrm{C} 6-\mathrm{C} 5-\mathrm{H} 5$ & 120.7 & $\mathrm{C} 18-\mathrm{C} 17-\mathrm{C} 9$ & $115.5(2)$ \\
\hline $\mathrm{C} 12-\mathrm{C} 5-\mathrm{H} 5$ & 120.7 & $\mathrm{C} 18-\mathrm{C} 17-\mathrm{H} 17 \mathrm{~A}$ & 108.4 \\
\hline $\mathrm{C} 5-\mathrm{C} 6-\mathrm{C} 7$ & $121.8(2)$ & C9-C17-H17A & 108.4 \\
\hline $\mathrm{C} 5-\mathrm{C} 6-\mathrm{H} 6$ & 119.1 & $\mathrm{C} 18-\mathrm{C} 17-\mathrm{H} 17 \mathrm{~B}$ & 108.4 \\
\hline $\mathrm{C} 7-\mathrm{C} 6-\mathrm{H} 6$ & 119.1 & $\mathrm{C} 9-\mathrm{C} 17-\mathrm{H} 17 \mathrm{~B}$ & 108.4 \\
\hline $\mathrm{C} 6-\mathrm{C} 7-\mathrm{C} 8$ & $119.9(3)$ & $\mathrm{H} 17 \mathrm{~A}-\mathrm{C} 17-\mathrm{H} 17 \mathrm{~B}$ & 107.5 \\
\hline $\mathrm{C} 6-\mathrm{C} 7-\mathrm{C} 16$ & $120.0(3)$ & $\mathrm{C} 17-\mathrm{C} 18-\mathrm{H} 18 \mathrm{~A}$ & 109.5 \\
\hline $\mathrm{C} 8-\mathrm{C} 7-\mathrm{C} 16$ & $120.1(3)$ & $\mathrm{C} 17-\mathrm{C} 18-\mathrm{H} 18 \mathrm{~B}$ & 109.5 \\
\hline $\mathrm{C} 13-\mathrm{C} 8-\mathrm{C} 7$ & $118.8(3)$ & $\mathrm{H} 18 \mathrm{~A}-\mathrm{C} 18-\mathrm{H} 18 \mathrm{~B}$ & 109.5 \\
\hline $\mathrm{C} 13-\mathrm{C} 8-\mathrm{H} 8$ & 120.6 & $\mathrm{C} 17-\mathrm{C} 18-\mathrm{H} 18 \mathrm{C}$ & 109.5 \\
\hline $\mathrm{C} 7-\mathrm{C} 8-\mathrm{H} 8$ & 120.6 & $\mathrm{H} 18 \mathrm{~A}-\mathrm{C} 18-\mathrm{H} 18 \mathrm{C}$ & 109.5 \\
\hline $\mathrm{C} 10-\mathrm{C} 9-\mathrm{C} 13$ & $100.8(2)$ & $\mathrm{H} 18 \mathrm{~B}-\mathrm{C} 18-\mathrm{H} 18 \mathrm{C}$ & 109.5 \\
\hline $\mathrm{C} 10-\mathrm{C} 9-\mathrm{C} 19$ & $111.4(2)$ & $\mathrm{C} 20-\mathrm{C} 19-\mathrm{C} 9$ & $116.0(2)$ \\
\hline $\mathrm{C} 13-\mathrm{C} 9-\mathrm{C} 19$ & $111.9(2)$ & $\mathrm{C} 20-\mathrm{C} 19-\mathrm{H} 19 \mathrm{~A}$ & 108.3 \\
\hline $\mathrm{C} 10-\mathrm{C} 9-\mathrm{C} 17$ & $112.0(2)$ & $\mathrm{C} 9-\mathrm{C} 19-\mathrm{H} 19 \mathrm{~A}$ & 108.3 \\
\hline $\mathrm{C} 13-\mathrm{C} 9-\mathrm{C} 17$ & $112.1(2)$ & $\mathrm{C} 20-\mathrm{C} 19-\mathrm{H} 19 \mathrm{~B}$ & 108.3 \\
\hline $\mathrm{C} 19-\mathrm{C} 9-\mathrm{C} 17$ & $108.6(2)$ & C9- $19-\mathrm{H} 19 \mathrm{~B}$ & 108.3 \\
\hline $\mathrm{C} 1-\mathrm{C} 10-\mathrm{C} 11$ & $122.2(2)$ & $\mathrm{H} 19 \mathrm{~A}-\mathrm{C} 19-\mathrm{H} 19 \mathrm{~B}$ & 107.4 \\
\hline $\mathrm{C} 1-\mathrm{C} 10-\mathrm{C} 9$ & $125.8(2)$ & $\mathrm{C} 19-\mathrm{C} 20-\mathrm{H} 20 \mathrm{~A}$ & 109.5 \\
\hline $\mathrm{C} 11-\mathrm{C} 10-\mathrm{C} 9$ & $112.0(2)$ & $\mathrm{C} 19-\mathrm{C} 20-\mathrm{H} 20 \mathrm{~B}$ & 109.5 \\
\hline $\mathrm{C} 10-\mathrm{C} 11-\mathrm{C} 4$ & $118.8(2)$ & $\mathrm{H} 20 \mathrm{~A}-\mathrm{C} 20-\mathrm{H} 20 \mathrm{~B}$ & 109.5 \\
\hline $\mathrm{C} 10-\mathrm{C} 11-\mathrm{C} 12$ & $107.6(2)$ & $\mathrm{C} 19-\mathrm{C} 20-\mathrm{H} 20 \mathrm{C}$ & 109.5 \\
\hline $\mathrm{C} 4-\mathrm{C} 11-\mathrm{C} 12$ & $133.6(2)$ & $\mathrm{H} 20 \mathrm{~A}-\mathrm{C} 20-\mathrm{H} 20 \mathrm{C}$ & 109.5 \\
\hline $\mathrm{C} 5-\mathrm{C} 12-\mathrm{C} 13$ & $119.8(2)$ & $\mathrm{H} 20 \mathrm{~B}-\mathrm{C} 20-\mathrm{H} 20 \mathrm{C}$ & 109.5 \\
\hline $\mathrm{C} 5-\mathrm{C} 12-\mathrm{C} 11$ & $132.4(2)$ & & \\
\hline $\mathrm{C} 10-\mathrm{C} 1-\mathrm{C} 2-\mathrm{C} 3$ & $-0.1(4)$ & $\mathrm{C} 10-\mathrm{C} 11-\mathrm{C} 12-\mathrm{C} 5$ & $180.0(3)$ \\
\hline
\end{tabular}




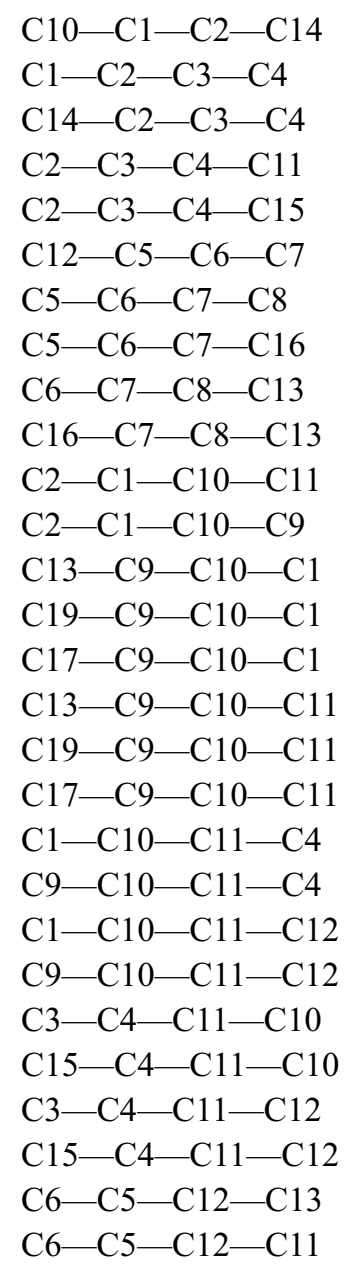

$-178.8(3)$
$-0.7(4)$
$178.0(3)$
$0.8(4)$
$179.6(3)$
$-1.3(5)$
$0.1(5)$
$-179.6(3)$
$0.9(5)$
$-179.4(3)$
$0.9(4)$
$-179.3(3)$
$-179.8(3)$
$61.4(3)$
$-60.5(4)$
$0.0(3)$
$-118.8(2)$
$119.3(3)$
$-0.8(4)$
$179.4(3)$
$179.7(3)$
$-0.1(3)$
$-0.1(4)$
$-178.6(3)$
$179.2(3)$
$0.7(5)$
$1.5(4)$
$-178.3(3)$

$0.6(6)$

$0.1(3)$

$-179.3(3)$

$-0.6(5)$

$178.7(3)$

$-0.6(4)$

$179.3(3)$

$-180.0(3)$

$-0.1(3)$

$-179.4(3)$

$-60.9(4)$

$61.4(4)$

$0.0(3)$

118.5 (3)

$-119.2(3)$

-175.8 (3)

2.9 (5)

-175.4 (3)

$3.2(5)$

-176.7 (4)

$3.5(6)$

$-57.6(3)$

54.8 (3)

179.0 (2)

56.6 (3)

$-55.3(3)$

$-179.6(2)$

Hydrogen-bond geometry $\left(A,{ }^{\circ}\right)$

\begin{tabular}{lllll}
\hline$D-\mathrm{H} \cdots A$ & $D-\mathrm{H}$ & $\mathrm{H} \cdots A$ & $D \cdots A$ & $D-\mathrm{H} \cdots A$ \\
\hline $\mathrm{C} 1-\mathrm{H} 1 \cdots \mathrm{O} 1^{\mathrm{i}}$ & 0.95 & 2.59 & $3.512(4)$ & 165 \\
$\mathrm{C} 5-\mathrm{H} 5 \cdots \mathrm{O} 2$ & 0.95 & 2.18 & $2.961(4)$ & 138 \\
$\mathrm{C} 5-\mathrm{H} 5 \cdots \mathrm{O} 3^{\mathrm{ii}}$ & 0.95 & 2.67 & $3.350(4)$ & 129 \\
$\mathrm{C} 16-\mathrm{H} 16 \cdots \mathrm{O} 2^{\mathrm{iii}}$ & 0.95 & 2.53 & $3.321(4)$ & 141 \\
$\mathrm{C} 17-\mathrm{H} 17 A \cdots \mathrm{O} 1^{\mathrm{i}}$ & 0.99 & 2.68 & $3.611(4)$ & 157 \\
\hline
\end{tabular}

Symmetry codes: (i) $-x+1,-y+1,-z+1$; (ii) $-x, y-1 / 2,-z-1 / 2$; (iii) $-x, y+1 / 2,-z-1 / 2$. 